\title{
One year with the EJNMMI
}

\section{Arturo Chiti ${ }^{1,2}$}

Published online: 10 March 2020

(C) Springer-Verlag GmbH Germany, part of Springer Nature 2020

More than one year has passed since I took over the editorship of our Journal. My predecessors, Peter Ell and Ignasi Carriò, made the EJNMMI a great journal. I know I am going to have a difficult time in keeping the same pace, but I will do what is in my best possibilities. At this time, I like to share some ideas on how to proceed in the next future.

The publishing world is evolving fast and we must be able to adapt to it. In this scenario, we appreciate the good reputation of our Journal and want to respect the principles of scientific integrity. News about citations factories, predatory journals, plagiarism, and fake news might give the general public a wrong impression on the reputation of all scientific Journals, including the EJNMMI. To protect the integrity and the reputation of our Journal is the responsibility of editors, reviewers, and authors. This is the only way we have to stand clear among the highly ranked Journals. Thanks to the clear policies and guidelines adopted by Springer Nature I can say that we did not face any serious issues in this context. When submitting or reviewing a paper, please pay the necessary attention to the Ethical statements required before publication. They are of paramount importance for the integrity of our Journal.

Fair competition and enthusiasm for science is what drives us to spend time, every day, to make EJNMMI a stronger Journal. We are the European Journal, but we represent more than European scientists. We play at a global level and compete in a global arena to attract the best papers in nuclear medicine, molecular imaging, theragnostic and radio

This article is part of the Topical Collection on Editorial

Arturo Chiti

arturo.chiti@hunimed.eu; cha@hunimed.onmicrosoft.com

1 Department of Biomedical Sciences, Humanitas University, Via Rita Levi Montalcini 4, 20090 Pieve Emanuele, Milan, Italy

2 Department of Diagnostic Imaging, Humanitas Clinical and Research Center - IRCCS, Rozzano, Italy pharmaceutical therapy. Good papers are supposed to generate citations that are supposed to be an indicator of a paper's impact on the scientific community. The European Journal of Nuclear Medicine and Molecular Imaging is in good shape and, besides some fluctuations in the impact factor, will keep on growing, thanks to your scientific contributions. The EJNMMI will stay as the home not only for good scientific papers but also for interesting and fruitful discussions among pairs on hot topics in our field. Recently, we appreciated interesting Letters, Editorials, and the first Interview. Some of them gave rise to controversial and will certainly keep the level of interest high for expert in the fields of our sciences.

We are all making an effort to make the EJNMMI more visible outside our field, nuclear medicine. This in not going to happen if we do not open our Journal to papers related to everything we are doing in our daily clinical practice and scientific research. Broadening the scope of our Journal does not mean to leave behind our pillars: nuclear medicine, molecular imaging, theragnostic and radiopharmaceutical therapy. On the contrary, it means to build a stronger reputation for our disciplines in the scientific world.

We have to invest more on our EJNMMI to make it a Journal appreciated by our clinical colleagues. We still have the tendency to send our best papers to clinical Journals and this has the consequence that the best Nuclear Medicine papers are, sometimes, not published on our Journal. The theoretical advantage is often translated into few points of impact factor; something that we would easy gain if we keep our best papers with our Journal. Think about an investment for the future of our discipline! Nowadays, readership is global, and our respected Colleagues would find your best paper over the web, not necessarily on the table of content of their Association's Journal. Investing on our Journal will have a great impact on the visibility of Nuclear Medicine, EANM and, of course, the whole EJNMMI family. 
Let me finish with a word of gratitude to authors and reviewers that are continuously supporting the Journal.

I am particularly grateful to Colleagues that never stopped contributing to the Journal from the beginning of the SARS$\mathrm{CoV}-2$ infection epidemics. In particular, those coming from Countries, first of all China, that are paying a great tribute to this difficult time. We work hard every day, we are fearless and we will not stop.

\section{Compliance with ethical standards}

Conflict of interest The author declares that there is no conflict of interest.
Ethics approval Not applicable

Publisher's note Springer Nature remains neutral with regard to jurisdictional claims in published maps and institutional affiliations. 